\title{
Influence of Pitch Size on Short-Term High Intensity Actions and Body Impacts in Soccer Sided Games
}

\author{
by \\ Daniel Castilloํㅜ Javier Raya-González ${ }^{1}$, Javier Yanci², Filipe Manuel Clemente ${ }^{3}$
}

\begin{abstract}
The aim of this study was to compare external training loads between small-sided games (SSGs) and largesided games (LSGs) in soccer players. Twenty outfield soccer players (14.8 \pm 0.6 years old) who competed in the Spanish U16 Provincial Division and belonged to the same team participated in the study. The soccer sided games were played at different individual interaction space (IIS) per player (i.e., SSG $=100 \mathrm{~m}^{2}$ and $L S G=200 \mathrm{~m}^{2}$ ) and were disputed in the same format (five-a-side plus goalkeepers) on two different pitch sizes (i.e., $38 \times 26$ vs. $53 \times 37 \mathrm{~m}$ ) defending an official soccer-goal. The sided games' duration was 4 bouts of 6 min with 2 min rest intervals between bouts. The results of this study showed no meaningful differences in the total distance and intensity of accelerations and decelerations between SSGs and LSGs except for the lower distance covered at medium intensity $\left(2.5-4 \mathrm{~m} \cdot \mathrm{s}^{-2}\right)$ observed during LSGs (-10.2\%; ES (effect size): -0.51). Players registered greater sprints, maximum velocity (Velmax) and body impacts at different intensities (i.e., I5-6g, I6-6.5g, I6.5-7g, I7-8g, I8-10g, in LSGs in comparison to SSGs. These findings suggest that an increase in the pitch size (i.e., IIS per player) can induce higher external loads for soccer players.
\end{abstract}

Key words: soccer, quantification, external loads, individual interaction space, neuromuscular.

\section{Introduction}

Soccer coaches usually use sided soccer games to periodize the weekly microcycle (Bujalance-Moreno et al., 2018) because these training drills can resemble the specific stimulus similar to real match-play in terms of the presence of teammates and opposition, rules, goalkeepers, score and limited duration (Castillo, RayaGonzalez, Weston et al., 2019). In addition, previous literature has demonstrated that the use of these training drills improves physical fitness of players (Bujalance-Moreno et al., 2018; Owen et al., 2012) as well as their technical and tactical abilities (Hammami et al., 2018). Considering that sided soccer games induce different physical responses, it could be relevant to quantify the external training loads to facilitate the development of specific training processes aimed at maximizing on-field performance (Mujika, 2013).

One of the main variables that influence external training loads during the development of sided soccer games is pitch size in terms of pitch proportions and goal objectives (Casamichana and Castellano, 2010; Kelly and Drust, 2009; López-Fernández et al., 2017). In this line, it is necessary to understand the individual interaction space (IIS), calculated by dividing the total pitch area by the number of players, considering that an ISS of approximately $300 \mathrm{~m}^{2}$ corresponds to each player during soccer match-play (Caro et al., 2019). Likewise, it is necessary to consider that the

\footnotetext{
1 - Faculty of Health Sciences, Universidad Isabel I, Burgos, Spain.

2 - Society, Sports and Physical Exercise Research Group (GIKAFIT), Physical Education and Sport Department, Faculty of Education and Sport, University of the Basque Country (UPV/EHU), Vitoria-Gasteiz, Spain.

3 - Escola Superior Desporto e Lazer, Instituto Politécnico de Viana do Castelo, Rua Escola Industrial e Comercial de Nun'Álvares, Viana do Castelo, Portugal.
} 
dynamics of the collective organization is conducive to smaller individual playing areas (82$94 \mathrm{~m}^{2}$ ) per player if one only considers the rectangle covering all outfield players during official matches (Fradua et al., 2013). Possibly because of this concept, previous studies have established that an IIS of around $100 \mathrm{~m}^{2}$ corresponds to small-sided games (SSGs), while an IIS of $200 \mathrm{~m}^{2}$ corresponds to large-sided games (LSGs) (Clemente et al., 2019; Owen et al., 2014; Sanchez-Sanchez et al., 2019). These aforementioned differences, focused on IIS, show a higher total running distance, high-intensity running and number of sprints during LSGs in comparison to SSGs (Casamichana and Castellano, 2010).

The ability of a player to perform highintensity actions over short distances (i.e., accelerations, decelerations and sprints) can be linked to common soccer-specific requirements such as tackling, players when in defense or to create space during possession, with sprints being the most frequent action preceding a goal (Buchheit et al., 2014; Cochrane and Monaghan, 2018; Mara et al., 2017). Researchers have shown that sided soccer games understimulate sprinting actions (Casamichana et al., 2012; Owen et al., 2014) and overstimulate short-term and highintensity actions (i.e., accelerations, decelerations, changes of direction) in comparison to match-play (Beenham et al., 2017; Casamichana et al., 2012; Clemente et al., 2019). Otherwise, research is inconclusive regarding the effect on the number and intensity of accelerations and decelerations when varying the pitch size during sided soccer games (Sanchez-Sanchez et al., 2019). While some authors have observed a higher number of accelerations and decelerations when a smaller IIS is implemented (Clemente et al., 2019), other researchers have shown greater accelerations and decelerations in sided soccer games contested in larger pitch sizes rather than smaller ones (Hodgson et al., 2014). The information is not only inconclusive regarding accelerations and decelerations when the IIS is varied, as no studies have analyzed how varying the pitch size affects the acceleration and deceleration intensities.

Another interesting variable to quantify during training sessions is body impact, which could provide relevant information regarding the mechanical stresses arising from the kinetic demands of all forces imposed on players during acceleration/deceleration, related changes of direction and impacts from both the player-toplayer collision and contact with the ground (Cummins et al., 2013; McLean et al., 2010; Vanrenterghem et al., 2017). In fact, body impacts have been demonstrated to be a useful variable to assess match-related fatigue (Russell et al., 2016), to understand training and match loads rather than other external loads (Abade et al., 2014; Arruda et al., 2015), to predict the training load measured by the rating of perceived moderate exertion (Gaudino et al., 2015), to provide specific soccer position profiles (Wellman et al., 2016) and to quantify the external load during sided soccer games. Thus, using body impacts as an external load variable would be interesting for coaches in order to widely understand the physical demands of the different soccer sided games.

The understanding of how sided games may be related to the conditions of physical impact, measured in the form of acceleration/deceleration intensities and body impacts, is a requirement to identify the best adjustments to make in order to standardize the stimulus to the player's needs. In fact, this is the necessary approach needed to qualify the physical demands, beyond just quantifying the extent of running by the players. Therefore, the aim of this study was to compare external training loads between small-sided games (SSGs) and largesided games (LSGs) in soccer players. We hypothesized that LSGs would induce greater external loads, caused by the pitch size, than SSGs.

\section{Methods}

\section{Participants}

Twenty outfield soccer players (age $=14.8$ \pm 0.6 years; body height $=173 \pm 7 \mathrm{~cm}$; body mass $=$ $60.6 \pm 8.1 \mathrm{~kg}$; training experience in the club $=3.8 \pm$ 2.6 years) who competed in the Spanish U16 Provincial Division and belonged to the same team participated in the study. A typical training session included a warm-up (general mobility games, technical-tactical drills), practice of specific game situations, small-sided games and practice of formal game situations (Sanchez-Sanchez et al., 2017). Each player was monitored four times in different sided games, thus a total of 80 observations were recorded for further analysis. 
All participants (i.e., 2 central defenders, 4 wingers, 2 midfielders and 2 forwards) trained for two hours, three days per week and were involved in official matches once per week, resulting in a total soccer practice time of $7.5 \mathrm{~h}$ per week. Players of the team with the most minutes played during matches and without injury in the month prior to the investigation were selected to participate. Coaches, players and parents or tutors were informed of the research procedures, requirements, benefits and potential risks before providing written informed consent. The study was performed in accordance with the Declaration of Helsinki (2013), approved by Ethics Committee under the code M10/2016/079.

\section{Procedures}

The study was conducted over a 2-week period at the middle of the competitive season. A previous week was used for familiarization with the study protocol including the use of Global Positional System (GPS) units and the same sided soccer games performed during the investigation period. The sided soccer games were played at different IIS: SSG $=100 \mathrm{~m}^{2}$ per player and LSG $=$ $200 \mathrm{~m}^{2}$ per player (Castellano et al., 2016). Each sided soccer game was performed twice with all games played within a period of 4 days, with an interval of $48 \mathrm{~h}$ after the last high load training session and/or official match, under similar weather conditions $\left(18-22 \mathrm{C}^{\circ}, 70-75 \%\right.$ humidity), on the same third-generation artificial pitch and with players wearing their normal soccer boots. Prior to the sided soccer games, players undertook a 20 min standardized warm-up, consisting of $7 \mathrm{~min}$ of slow jogging and strolling locomotion followed by $10 \mathrm{~min}$ of specific soccer drills, finishing with $3 \mathrm{~min}$ of progressive sprints and accelerations. During the study, players and their parents were instructed to maintain their usual habits, which included $8 \mathrm{~h}$ of night-time sleep before each data collection session, and adequate hydration and carbohydrate intake over the $24 \mathrm{~h}$ prior to each experimental sided game (Sanchez-Sanchez et al., 2018).

Sided soccer games

Each SSG and LSG was contested in the same format (five-a-side plus goalkeepers) on two different pitch sizes (i.e., IIS of $100 \mathrm{~m}^{2}$ per player $=$ $38 \times 26 \mathrm{~m}$ vs. IIS of $200 \mathrm{~m}^{2}$ per player $=53 \times 37 \mathrm{~m}$ ) playing with directionality and with the implications of the soccer rules. The sided soccer games' duration was 4 bouts of 6 min each with a 2 min rest interval between bouts. Although sided soccer games were played on different IIS (i.e., 100 vs. $200 \mathrm{~m}^{2}$ per player), the relative pitch proportions were held constant with the same ratio (i.e., length/width $=1.46$ ) as a soccer match. The teams were organized according to playing positions (i.e., one goalkeeper, one central defender, two wingers, one midfielder and one forward), technical-tactical levels, competitive experience, aerobic fitness and coach qualitative evaluation and they were faced all times (Casamichana and Castellano, 2010). The teams were the same (including the same players) during each experimental protocol. Coaches did not provide any strategic or tactical feedback during the process. Only verbal encouragement was provided to ensure a high commitment level of players during the matches. Goalkeepers were not included in further analyses due to their specific role. In addition, minor rule modifications were applied, such as restarting the game after a goal by the goalkeeper and kick-ins awarded to the opposing side from that of the player who last touched the ball (Rebelo et al., 2016). When the ball left the playing field, the coach always reintroduced balls immediately during all the sided soccer games (Owen et al., 2012). We respected the off-side rule with the aim of not changing the real structure of the game.

Measures

Measures were assessed with GPS devices (WIMU PROTM, RealTrack Systems, Almería, Spain) operating at a sampling frequency of $10 \mathrm{~Hz}$ (Muñoz-Lopez et al., 2017). Microsensor units were harnessed within a tight-fitting vest that was worn by soccer players during the study. The microsensor devices were activated $15 \mathrm{~min}$ before the start of each testing session, in accordance with the manufacturer's recommendations. Data were downloaded post-intervention protocol to a computer and analyzed using a customized software package (WIMU SPRO, Almería, Spain). The total distance covered while accelerating (Acc) and decelerating (Dec) was taken as a key outcome measures with further distance measures derived for different intensity categories (Castillo, Rodriguez-Fernandez et al., 2019): low-intensity acceleration (LAcc; $1-2.5 \mathrm{~m} \cdot \mathrm{s}^{-2}$ ), medium-intensity acceleration (MAcc; 2.5-4 $\mathrm{m} \cdot \mathrm{s}^{-2}$ ), high-intensity acceleration (HAcc; $>4 \quad \mathrm{~m} \cdot \mathrm{s}^{-2}$ ), low-intensity 
deceleration (LDec; $-1 /-2.5 \quad \mathrm{~m} \cdot \mathrm{s}^{-2}$ ), mediumintensity deceleration (MDec; $-2.5 /-4 \mathrm{~m} \cdot \mathrm{s}^{-2}$ ), and high-intensity deceleration (HDec; $<-4 \mathrm{~m} \cdot \mathrm{s}^{-2}$ ). The number of sprints $\left(>21 \mathrm{~km} \cdot \mathrm{h}^{-1}\right)$ and the maximum velocity (Velmax) achieved were registered as indicators of the external load (Castillo et al., 2016). In addition, the GPS devices were coupled with a $100 \mathrm{~Hz}$ triaxial accelerometer that allowed for the estimation of body impact data provided in "g" force. An impact was counted by the system if the force applied was more than five $g$ force units $(5 \mathrm{~g})$. The software displayed the total impact counts from collisions, the intensity, as well as the time during the game or training drill where the impact occurred. A scaling system between $5-10+\mathrm{g}$ for grading the impacts (I) was used: I5-6g: light impact, hard acceleration/deceleration/change of direction; I66.5g: light to moderate impact (player collision, contact with the ground); I6.5-7g: moderate to heavy impact; I7-8g: heavy impact; I8-10g: very heavy impact (scrum engagement); and $\mathrm{I} 10+\mathrm{g}$ : severe impact/tackle/collision. Impacts above $10 \mathrm{~g}$ were used to report on the number of severe impacts that the players received during games (Wellman et al., 2016). The equipment presented good levels of validity and reliability in linear, circular and zig-zag courses (Bastida-Castillo et al., 2018; Muñoz-Lopez et al., 2017). Data were collected during what were considered to be good weather and satellite conditions for the GPS (number of satellites $=10.1 \pm 0.2$ ).

\section{Statistical analysis}

Results are presented as mean \pm standard deviations (SD). Normal distribution and homogeneity of variances were tested using the Shapiro-Wilk and Levene tests. All analyzed variables had a normal distribution, and parametric techniques were applied. A t-test for paired samples was used to analyze the differences in external responses between SSGs and LSGs. Statistical significance was set at $p<$ 0.05. Data analysis was performed using the Statistical Package for Social Sciences (version 21.0 for Windows, SPSS Inc, Chicago, IL, USA). We elected to use effect sizes (ES), with uncertainty of the estimates shown as $90 \%$ confidence limits (CL), to quantify the magnitude of the differences between playing in SSGs (i.e., $100 \mathrm{~m}^{2}$ per player) and LSGs (i.e., $200 \mathrm{~m}^{2}$ per player) in external load variables (i.e., Acc, Dec, LAcc, MAcc, HAcc, LDec,
MDec, HDec, Velmax, sprints and number and intensities of body impacts). The ES was classified as trivial $(<0.2)$, small $(0.2-0.6)$, moderate $(0.6-1.2)$, large (1.2-2.0), very large (2.0-4.0) and extremely large (>4.0) (Hopkins et al., 2009). A threshold value of 0.2 between-subject standard deviations was set as the smallest worthwhile change, and inference was then based on the disposition of the $\mathrm{CL}$ for the mean difference to this smallest worthwhile effect; the probability (percentage chances) that the true difference between tests is substantial (beneficial/detrimental) or trivial was calculated as per the magnitude-based inference (MBI) approach (Batterham and Hopkins, 2006). The percentage chances were then qualified via probabilistic terms and assigned using the following scale: $25-75 \%$, possibly; $75-95 \%$, likely; 95-99.5\%, very likely; $>99.5 \%$, most likely (Hopkins et al., 2009). Inference was classified as unclear if the $90 \%$ CL overlapped the thresholds for the smallest worthwhile positive and negative effects (Hopkins et al., 2009). Given the relatively small size of our study, in both the number of players and the number of sessions, we employed a conservative approach to inference whereby only ES $\geq$ moderate was deemed meaningful.

\section{Results}

No significant differences $(p>0.05$; unclear to possibly small) were found in the total distance and intensities of accelerating and decelerating between SSGs and LSGs (Table 1).

Players completed a greater number of sprints $(p<0.001$; most likely very large) and achieved higher Velmax $(p<0.001$; most likely large) in LSGs in comparison with SSGs (Figure 1).

Players were exposed to greater $(p<0.001$; very likely moderate) body impacts in LSGs compared to SSGs. Also, players registered more body impacts at I5-6g intensity in LSGs than in SSGs $(p<0.001$; most likely moderate to likely small). However, no significant differences $(p>$ 0.05 ) were found at I6-6.5g, I-6.7g, I7-8g, 8-10g and $\mathrm{I}>10+\mathrm{g}$ between the mentioned pitch sizes. 
Table 1

Results (mean differences $\pm S D$ ) of the number and intensity of accelerations and decelerations between small-sided games (SSGs) and large-sided games (LSGs) along with effect sizes (ES) and qualitative inferences

\begin{tabular}{|c|c|c|c|c|c|c|c|}
\hline Variables & SSG & LSG & $\begin{array}{c}\text { Mean } \\
\text { difference; } \\
\pm 90 \% \mathrm{CL}\end{array}$ & $p$ & $\mathrm{ES} ; \pm 90 \% \mathrm{CL}$ & $\begin{array}{l}\text { Qualitative } \\
\text { Inference }\end{array}$ & Rating \\
\hline $\operatorname{Acc}(\mathrm{m})$ & $409.40 \pm 47.11$ & $402.70 \pm 57.12$ & $-2.0 ; \pm 4.7$ & 0.56 & $-0.17 ; \pm 0.41$ & Unclear & $7 / 47 / 46$ \\
\hline $\operatorname{Dec}(m)$ & $352.55 \pm 51.63$ & $360.70 \pm 53.13$ & $2.4 ; \pm 4.7$ & 0.38 & $0.15 ; \pm 0.29$ & Possibly Trivial & $39 / 59 / 3$ \\
\hline $\operatorname{LAcc}(\mathrm{m})$ & $307.25 \pm 41.71$ & $310.10 \pm 50.64$ & $0.4 ; \pm 5.1$ & 0.75 & $0.03 ; \pm 0.37$ & Unclear & $22 / 63 / 15$ \\
\hline $\operatorname{MAcc}(\mathrm{m})$ & $88.10 \pm 18.32$ & $79.35 \pm 15.35$ & $-10.2 ; \pm 8.4$ & 0.06 & $-0.51 ; \pm 0.44$ & Likely Small & $1 / 11 / 88$ \\
\hline $\operatorname{HAcc}(\mathrm{m})$ & $14.05 \pm 6.81$ & $13.25 \pm 6.86$ & $-4.6 ; \pm 21.4$ & 0.54 & $-0.08 ; \pm 0.40$ & Unclear & $12 / 57 / 31$ \\
\hline $\operatorname{LDec}(\mathrm{m})$ & $266.90 \pm 40.31$ & $277.05 \pm 47.13$ & $3.4 ; \pm 5.2$ & 0.20 & $0.21 ; \pm 0.31$ & Possibly Small & $52 / 46 / 2$ \\
\hline $\operatorname{MDec}(\mathrm{m})$ & $70.30 \pm 13.07$ & $66.75 \pm 12.73$ & $-4.8 ; \pm 7.7$ & 0.22 & $-0.25 ; \pm 0.41$ & Possibly Small & $4 / 38 / 58$ \\
\hline $\operatorname{HDec}(\mathrm{m})$ & $15.35 \pm 5.91$ & $16.90 \pm 5.87$ & $11.0 ; \pm 19.3$ & 0.29 & $0.27 ; \pm 0.45$ & Possibly Small & $61 / 35 / 4$ \\
\hline
\end{tabular}

$C L=$ confident limits; $S D=$ standard deviation; $A c c=$ total distance covered accelerating; $D e c=$ total distance covered decelerating; $L A c c=$ distance covered accelerating between 1 and $2.5 \mathrm{~m} \cdot \mathrm{s}^{-2} ; M A c c=$ distance covered accelerating between 2.5 and $4 \mathrm{~m} \cdot \mathrm{s}^{-2} ; \mathrm{HAcc}=$ distance covered accelerating at above $4 \mathrm{~m} \cdot \mathrm{s}^{-2} ; \mathrm{LDec}=$ distance covered decelerating between -2.5 and $-1 \mathrm{~m} \cdot \mathrm{s}^{-2} ; \mathrm{MDec}=$ distance covered decelerating between -4 and $-2.5 \mathrm{~m} \cdot \mathrm{s}^{-}$ 2; HDec $=$ distance covered decelerating at less than $-4 \mathrm{~m} \cdot \mathrm{s}^{-2}$

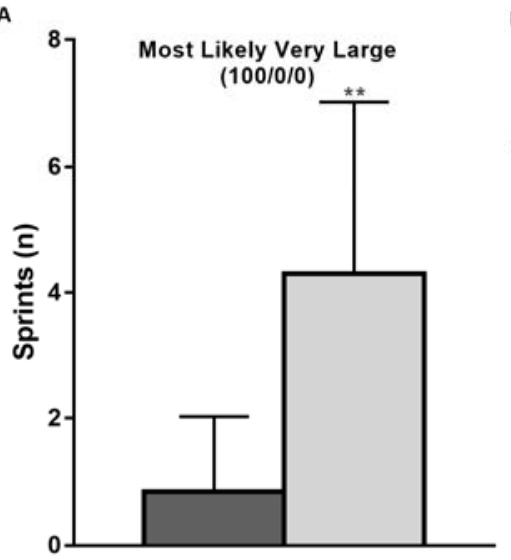

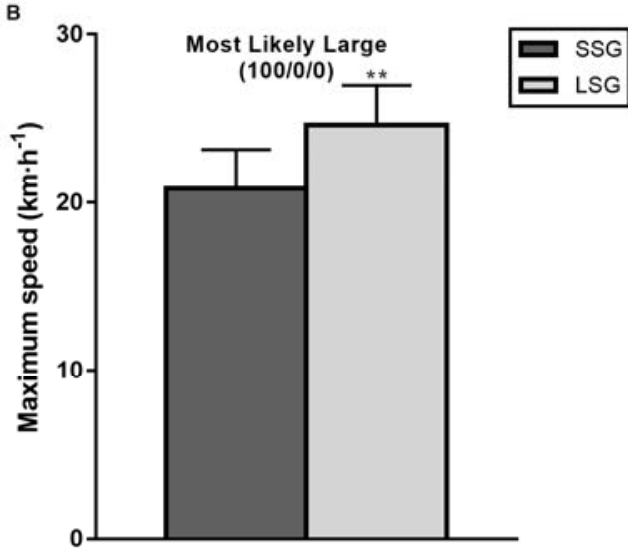

Figure 1

** Significant level set at $p<0.001$

Practical differences in sprints number $(A)$ and maximum speed $(B)$ between small-sided game (SSG) and large-sided game (LSG). 


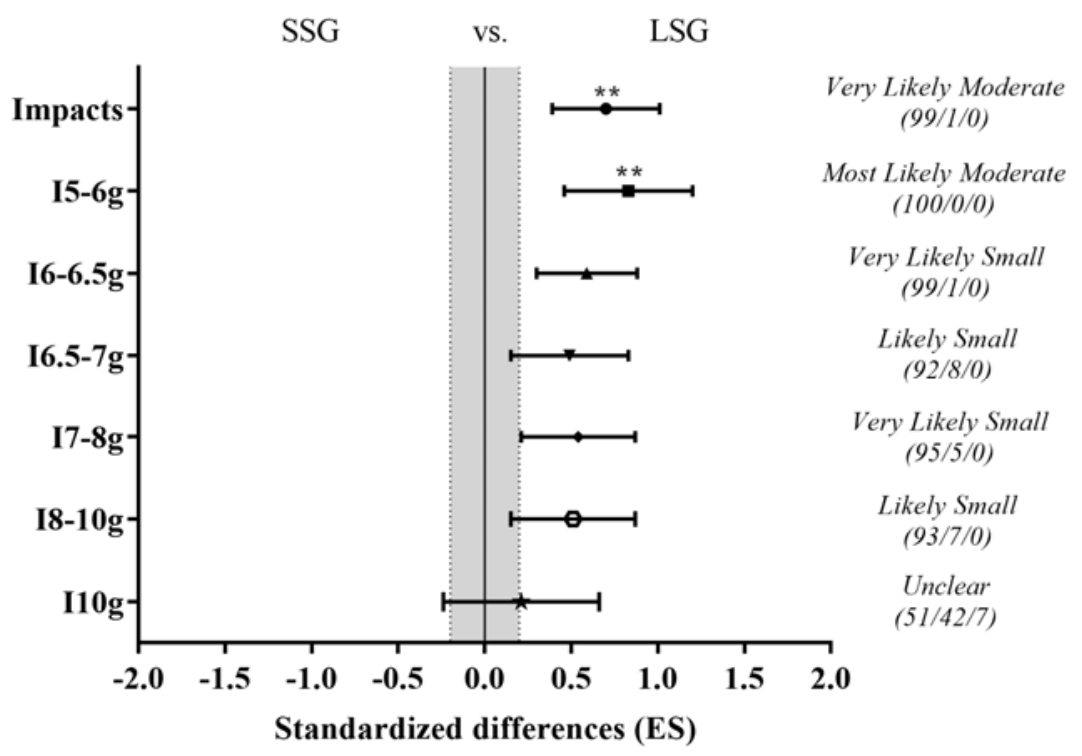

Figure 2

Practical differences between small-sided game (SSG) and large-sided game (LSG) in impact variables. Impacts = total impacts; I5-6g = light impact, hard acceleration/deceleration/ change of direction; I6$6.5 \mathrm{~g}=$ light to moderate impact (player collision, contact with the ground); $16.5-7 \mathrm{~g}=$ moderate to heavy impact; $17-8 \mathrm{~g}=$ heavy impact; $18-10 \mathrm{~g}=$ very heavy impact (scrum engagement); $>I 10 \mathrm{~g}=$ severe impact/ tackle/ collision.

** Significant level set at $p<0.001$

\section{Discussion}

The aim of this study was to compare external training loads between SSGs and LSGs in soccer players. This is the first study which has provided information about ranges of short-term and high-intensity actions depending on the pitch size in sided soccer games. The results of this investigation showed that although no substantial differences were present in total distance and intensities for accelerating and decelerating between SSGs and LSGs, players registered a greater number of sprints, higher Velmax and a greater number of body impacts and body impacts at $\mathrm{I5}-6 \mathrm{~g}$ intensity during LSGs in comparison to SSGs.

Short-term and high-intensity actions have been demonstrated to be decisive in soccer, as these actions may have a specific impact on the outcome of the matches and physical performance of players (Cochrane and Monaghan, 2018). The literature has shown contradictory results regarding the number of accelerations and decelerations when coaches use different pitch sizes. On the one hand, higher acceleration sums were reported in SSGs (i.e., IIS $=120 \mathrm{~m} 2$ per player) as compared to LSGs (i.e., $194 \mathrm{~m} 2$ per player) (Clemente et al., 2019). On the other hand, Hodgson et al. (2014) observed greater distances for accelerations (SSGs $=230111$ vs. LSGs $=327$

$70 \mathrm{~m}$ ) and decelerations (SSGs $=198 \quad 89$ vs. LSGs $=29868 \mathrm{~m}$ ) in LSGs (i.e., IIS $=200 \mathrm{~m} 2$ ) than in SSGs (i.e., IIS $=60 \mathrm{~m} 2$ ). In addition, these authors also reported greater distances for 
accelerating and decelerating at low, moderate and high intensities (Hodgson et al., 2014). However, our results do not coincide with any of these previous studies as no significant differences were found in the distance covered at different intensities of accelerations and decelerations between SSGs and LSGs. These differences could be due to the characteristics of sided games in terms of duration, the off-side rule, presence of goalkeepers, the number of players and IIS. Therefore, further studies focused on the influence of pitch size on accelerations and decelerations should be developed to clarify the aforementioned controversy.

In addition to the accelerations and decelerations covered at different intensities, it has been demonstrated that the analysis of sprinting actions in soccer is crucial to optimize performance, mainly due to the fact that sprinting is the most frequent action preceding a goal (Faude et al., 2012). Thus, understanding the sprinting demands of each training drill in order to replicate them during training periodization is an important challenge of soccer coaches. In this sense, our results are consistent with previous studies which showed that a greater number of sprints and Velmax values are achieved during LSGs than during SSGs (Casamichana and Castellano, 2010; Clemente et al., 2019; Hodgson et al., 2014). In this sense, it seems that an increase of the pitch size, holding constant the relative pitch proportions with the same ratio (i.e., length/width $=1.46$ ) as a soccer match, would be a useful strategy to enhance the number of sprints and the Velmax achieved by young soccer players. However, the number of sprints registered in the present study in sided soccer games played with a higher IIS per player (i.e., LSGs) was lower ( 4 vs. 10 sprints) than observed in other studies (Clemente et al., 2019; Hodgson et al., 2014), mainly due to the inclusion of the off-side rule and the age-group effect. Considering these differences, it seems that sprinting actions and Velmax may not be influenced only by the ISS per player, but also by other decisive factors such as the pitch proportions, the number of players and/or the modification of some rules of the game (i.e., offside). The acquired knowledge of the external loads imposed upon players during sided soccer games in relation to pitch size could be interesting for coaches in order to understand the impact of sided soccer games.

Despite the scarce literature focused on body impacts in soccer, it seems that the quantification of this variable could be interesting in order to implement appropriate training strategies (Abade et al., 2014; Arruda et al., 2015). To date, although some authors have reported information about the number and intensities of body impacts during official soccer games (Russell et al., 2016; Wellman et al., 2016), only one study has provided data during training drills (Castillo, Raya-Gonzalez, Clemente et al., 2019). In line with this investigation, the results of our study showed that greater body impacts were registered in LSGs compared to SSGs. In addition, players registered higher body impacts at $15-6 \mathrm{~g}$ intensity in LSGs rather than SSGs. Thus, it seems that playing at a higher ISS per player induces greater body impacts. In this sense, it would be advisable to quantify the physiological and neuromuscular effects caused by the body impacts experienced by players during sided soccer games (Arruda et al., 2015; Wellman et al., 2016). Further research should investigate whether higher body impacts during training drills can induce training-related neuromuscular fatigue or risk of injury, or on the contrary, whether this variable can be beneficial to enhance chronic adaptations for maximizing the players' physical performance. Given that differences have been found in body impacts, but no differences were reported regarding accelerations and decelerations, it would be convenient to quantify both variables during specific training drills in order to acquire greater knowledge of the sided soccer game demands. Further research could investigate the association of body impacts with other external loads. These findings provide novel quantification of sided soccer games and may indicate that body impacts can be used to increase the specificity of training programs.

This study is not without limitations, with the main one being a small sample size. Besides, understanding the soccer games-related fatigue as previous studies have analyzed (Castillo, Rodriguez-Fernandez et al., 2019; Rebelo et al., 2016), it would be interesting for further investigations to extend the knowledge of the training process including this information. In addition, considering the inclusion of the offside 
rule during the soccer sided games, this fact could influence the dynamics of the game, so it would be advisable to replicate this study without the application of the offside. Finally, for future investigations, it would be interesting to determine the characteristics of body impacts encountered during match-play in order to organize the weekly loads and to inspect the relationships between tactical behaviors and collective patterns of play both in youth and senior soccer players.

\section{Conclusions}

While no meaningful differences were found in the total distance and intensities during accelerations and decelerations between SSGs and LSGs, a greater number of sprints, Velmax and body impacts were performed during LSGs in comparison with SSGs. These results suggest that an increase in the pitch size (i.e., IIS per player) can induce higher external loads for the soccer player. These findings provide insightful knowledge that can be used to design specific training drills in soccer, and that the quantification of body impacts could be used to increase the specificity of training programs.

\section{Acknowledgments}

The authors gratefully acknowledge the support of a Spanish government subproject Mixed method approach on performance analysis (in training and competition) in elite and academy sport [PGC2018098742-B-C33] (2019-2021), from Ministerio de Ciencia, Innovación y Universidades (MCIU), Agencia Estatal de Investigación (AEI), and Fondo Europeo de Desarrollo Regional (FEDER), that is part of the coordinated project New approach of research in physical activity and sport from mixed methods perspective (NARPAS_MM) [SPGC201800X098742CV0].

\section{References}

Abade EA, Gonçalves BV, Leite NM, Sampaio JE. Time-motion and physiological profile of football training sessions performed by Under-15, Under-17, and Under-19 elite Portuguese players. Int J Sports Physiol Perform, 2014; 9(3): 463-470

Arruda AFS, Carling C, Zanetti V, Aoki MS, Coutts AJ, Moreira A. Effects of a very congested match schedule on body-load impacts, accelerations, and running measures in youth soccer players. Int J Sports Physiol Perform, 2015; 10(2): 248-252

Bastida-Castillo A, Gómez-Carmona CD, De la Cruz-Sánchez E, Pino-Ortega J. Accuracy, intra- and interunit reliability, and comparison between GPS and UWB-based position-tracking systems used for time-motion analyses in soccer. Eur J Sport Sci, 2018; 18(4): 450-457

Batterham AM, Hopkins WG. Making meaningful inferences about magnitudes. Int J Sports Physiol Perform, 2006; 1(1): 50-57

Beenham M, Barron DJ, Fry J, Hurst HH, Figueirdo A, Atkins S. A comparison of GPS workload demands in match play and small-sided games by the positional role in youth soccer. J Hum Kinet, 2017; 57(1): 129137

Buchheit M, Haddad H Al, Simpson BM, et al. Monitoring accelerations with gps in football: Time to slow down. Int J Sports Physiol Perform, 2014; 9(3): 442-445

Bujalance-Moreno P, Garcia-Pinillos F, Latorre-Roman PA. Effects of a small-sided game-based training program on repeated sprint and change of direction abilities in recreationally-trained soccer players. $J$ Sports Med Phys Fitness, 2018; 58(7-8): 1021-1028

Bujalance-Moreno P, Latorre-Roman PA, Garcia-Pinillos F. A systematic review on small-sided games in football players: Acute and chronic adaptations. J Sports Sci, 2018: 1-29. doi:10.1080/02640414.2018.1535821

Caro O, Zubillaga A, Fradua L, Fernandez-Navarro J. Analysis of playing area dimensions in Spanish professional soccer: extrapolation to the design of small-sided games with tactical applications. $J$ Strength Cond Res, 2019. doi:10.1519/JSC.0000000000003226 
Casamichana D, Castellano J. Time-motion, heart rate, perceptual and motor behaviour demands in smallsides soccer games: Effects of pitch size. J Sports Sci, 2010; 28(14): 1615-1623

Casamichana D, Castellano J, Castagna C. Comparing the physical demands of friendly matches and smallsided games in semiprofessional soccer players. J Strength Cond Res, 2012; 26(3): 837-843

Castellano J, Puente A, Echeazarra I, Usabiaga O, Casamichana D. Number of players and relative pitch area per player: comparing their influence on heart rate and physical demands in under-12 and under-13 football players. Plos One, 2016; 11(1): e0127505

Castillo D, Cámara J, Castellano J, Yanci J. Match officials do not attain maximal sprinting speed during matches. Kinesiol, 2016; 48(2): 207-212

Castillo D, Raya-Gonzalez J, Weston M, Yanci J. Distribution of external load during acquisition training sessions and match play of a proofessional soccer team. J Strength Cond Res, 2019. doi: 10.1519/JSC.0000000000003363

Castillo D, Raya-González J, Clemente FM, Yanci J. The influence of youth soccer players' sprint performance on the different sided games' external load using GPS devices. Res Sports Med, 2019. doi:10.1080/15438627.2019.1643726

Castillo D, Rodriguez-Fernandez A, Nakamura FY, et al. (2019). Influence of different small-sided game formats on physical and physiological demands and physical performance in young soccer players. $J$ Strength Cond Res, 2019. doi:10.1519/JSC.0000000000003114

Clemente FM, Sarmento H, Rabbani A, Van Der Linden CMIN, Kargarfard M, Costa IT. Variations of external load variables between medium- and large-sided soccer games in professional players. Res Sports Med, 2019; 27(9): 50-59

Cochrane DJ, Monaghan D. Using sprint velocity decrement to enhance acute sprint performance. J Strength Cond Res, 2018. doi:10.1519/JSC.0000000000002707

Cummins C, Orr R, O'Connor H, West C. Global positioning systems (GPS) and microtechnology sensors in team sports: a systematic review. Sports Med, 2013; 43(10): 1025-1042

Faude O, Koch T, Meyer T. Straight sprinting is the most frequent action in goal situations in professional football. J Sports Sci, 2012; 30(7): 625-631

Fradua L, Zubillaga A, Caro O, Ivan Fernandez-Garcia A, Ruiz-Ruiz C, Tenga A. Designing small-sided games for training tactical aspects in soccer: extrapolating pitch sizes from full-size professional matches. J Sports Sci, 2013; 31(6): 573-581

Gaudino P, Iaia FM, Strudwick AJ, Hawkins DH, Alberti G, Atkinson G, Gregson W. Factors influencing perception of effort (session rating of perceived exertion) during elite soccer training. Int J Sport Physiol Perform, 2015; 10(7): 860-864

Hammami A, Gabbett TJ, Slimani M, Bouhlel E. Does small-sided games training improve physical-fitness and specific skills for team sports? A systematic review with meta-analysis. J Sports Med Phys Fitness, 2018; 58(10): 1446-1455

Hodgson C, Akenhead R, Thomas K. Time-motion analysis of acceleration demands of 4v4 small-sided soccer games played on different pitch sizes. Hum Mov Sci, 2014; 33: 25-32

Hopkins WG, Marshall SW, Batterham AM, Hanin J. Progressive statistics for studies in sports medicine and exercise science. Med Sci Sport Exerc, 2009; 41(1): 3-13

Kelly DM, Drust B. The effect of pitch dimensions on heart rate responses and technical demands of smallsided soccer games in elite players. J Sci Med Sport, 2009; 12(4): 475-479

López-Fernández J, Gallardo L, Fernández-Luna Á, Villacañas V, García-Unanue J, Sánchez-Sánchez J. Pitch size and game surface in different small-sided games. Global indicators, activity profile and acceleration of female soccer players. J Strength Cond Res, 2017. doi: 10.1519/JSC.0000000000002090

Mara JK, Thompson KG, Pumpa KL, Morgan S. The acceleration and deceleration profiles of elite female soccer players during competitive matches. J Sci Med Sport, 2017; 20(9): 867-872

McLean BD, Coutts AJ, Kelly V, McGuigan MR, Cormack SJ. Neuromuscular, endocrine, and perceptual fatigue responses during different length between-match microcycles in professional rugby league players. Int J Sport Physiol Perform, 2010; 5(3): 367-383

Mujika I. The alphabet of sport science research starts with Q. Int J Sport Physiol Perform, 2013; 8(5): 465-466 
Muñoz-Lopez A, Granero-Gil P, Pino-Ortega J, De Hoyo M. The validity and reliability of a 5-hz GPS device for quantifying athletes' sprints and movement demands specific to team sports. J Hum Sport Exerc, 2017; 12(1): 156-166

Owen AL, Wong DP, Paul D, Dellal A. Physical and technical comparisons between various-sided games within professional soccer. Int J Sports Med, 2014; 35(4): 286-292. doi:10.1055/s-0033-1351333

Owen AL, Wong DP, Paul D, Dellal A. Effects of a periodized small-sided game training intervention on physical performance in elite professional soccer. J Strength Cond Res, 2012; 26(10): 2748-2754

Rebelo AN, Silva P, Rago V, Barreira D, Krustrup P. Differences in strength and speed demands between $4 \mathrm{v} 4$ and 8v8 small-sided football games. J Sport Sci, 2016; 34: 2246-2254

Russell M, Sparkes W, Northeast J, Cook CJ, Love TD, Bracken RM, Kilduff LP. Changes in acceleration and deceleration capacity throughout professional soccer match-play. J Strength Cond Res, 2016; 30(10): 2839-2844

Sanchez-Sanchez J, Ramirez-Campillo R, Carretero M, Martín V, Hernández D, Nakamura FY. Soccer smallsided games activities vary according to the interval regime and their order of presentation within the session. J Hum Kinet, 2018; 62: 167-175

Sanchez-Sanchez J, Sanchez M, Hernández D, Gonzalo-Skok O, Casamichana D, Ramirez-Campillo R, Nakamura FY. Physical performance during soccer-7 competition and small-sided games in U12 players. J Hum Kinet, 2019; 67: 281-290

Sanchez-Sanchez J, Hernández D, Casamichana D, Martinez-Salazar D, Ramirez-Campillo R, Sampaio J. Heart rate, technical performance, and session-RPE in elite youth soccer small-sided games played with wildcard players. J Strength Cond Res, 2017; 31(10): 2678-2685

Vanrenterghem J, Nedergaard NJ, Robinson MA, Drust B. Training load monitoring in team sports: A novel framework separating physiological and biomechanical load-adaptation pathways. Sports Med, 2017; 47(11): 2135-2142

Wellman AD, Coad SC, Goulet GC, Coffey VG, McLellan CP. Quantification of accelerometer derived impacts associated with competitive games in NCAA Division I College Football players. J Strength Cond Res, 2016; 31(2): 1

\section{Corresponding author:}

\section{Dr. Javier Raya-González}

Faculty of Health Sciences, Universidad Isabel I.

Fernán González, 76, 09003, Burgos, Spain

Telephone number: +0034 947671731.

E-mail address: rayagonzalezjavier@gmail.com 